\title{
DT-Web: a web-based application for drug-target interaction and drug combination prediction through domain-tuned network-based inference
}

Salvatore Alaimo ${ }^{1}$, Vincenzo Bonnici ${ }^{3}$, Damiano Cancemi ${ }^{1}$, Alfredo Ferro ${ }^{2}$, Rosalba Giugno ${ }^{2,4^{*}+}$, Alfredo Pulvirenti ${ }^{2^{*}+}$

From Eleventh Annual Meeting of the Bioinformatics Italian Society Meeting

Rome, Italy. 26-28 February 2014

\begin{abstract}
Background: The identification of drug-target interactions (DTI) is a costly and time-consuming step in drug discovery and design. Computational methods capable of predicting reliable DTI play an important role in the field. Algorithms may aim to design new therapies based on a single approved drug or a combination of them. Recently, recommendation methods relying on network-based inference in connection with knowledge coming from the specific domain have been proposed.

Description: Here we propose a web-based interface to the DT-Hybrid algorithm, which applies a recommendation technique based on bipartite network projection implementing resources transfer within the network. This technique combined with domain-specific knowledge expressing drugs and targets similarity is used to compute recommendations for each drug. Our web interface allows the users: (i) to browse all the predictions inferred by the algorithm; (ii) to upload their custom data on which they wish to obtain a prediction through a DT-Hybrid based pipeline; (iii) to help in the early stages of drug combinations, repositioning, substitution, or resistance studies by finding drugs that can act simultaneously on multiple targets in a multi-pathway environment. Our system is periodically synchronized with DrugBank and updated accordingly. The website is free, open to all users, and available at http://alpha.dmi.unict.it/dtweb/.

Conclusions: Our web interface allows users to search and visualize information on drugs and targets eventually providing their own data to compute a list of predictions. The user can visualize information about the characteristics of each drug, a list of predicted and validated targets, associated enzymes and transporters. A table containing key information and GO classification allows the users to perform their own analysis on our data. A special interface for data submission allows the execution of a pipeline, based on DT-Hybrid, predicting new targets with the corresponding p-values expressing the reliability of each group of predictions. Finally, It is also possible to specify a list of genes tracking down all the drugs that may have an indirect influence on them based on a multi-drug, multi-target, multi-pathway analysis, which aims to discover drugs for future follow-up studies.
\end{abstract}

\footnotetext{
* Correspondence: apulvirenti@dmi.unict.it

† Contributed equally

${ }^{2}$ Department of Clinical and Experimental Medicine, University of Catania,

Viale A. Doria 6, Catania, Italy

Full list of author information is available at the end of the article
}

(C) 2015 Alaimo et al.; licensee BioMed Central Ltd. This is an Open Access article distributed under the terms of the Creative Commons 


\section{Background}

In the last decades, pharmacology and therapeutic fields have encountered several development shortcomings due to prohibitive clinical costs related to novel drug discovery. The development of a new molecular entity is typically based on the process of discovering a new drug by modifying an existing one [1]. Recent trends in the pharmacogenomics area are going to exploit data mining and bioinformatics approaches, such as those based on drugs similarity, in connection with biological networks analysis. In Phatak et al. [2], a computational method for drug repositioning differs from previous similarity-based approaches since it combines chemical drug structures and drug target information computing similarity of drug target profiles via a bipartite-graph based approach. Bipartite graphs can also be used to provide a drug-target network for assessing the similarity between different disease inhibitors based on the connection to other compounds and targets. In this case, classical structure-based drug design and chemicalgenomic similarity methods are combined with molecular graph theories. Another example of network based drug study is shown in Iorio et al. [3], where drug mode of action and drug repositioning are assessed using available gene expression profiles [4] to build a drugdrug network. The Connectivity Map databases [4] is a comprehensive reference catalog of genome-wide expression data from cultured human cells perturbed with many chemicals and genetic reagents, to connect human diseases with the genes that may cause them and drugs that can treat them. Literature-mining studies show that a large majority of new drugs bind to targets in some way related to a previously existing one [5-7]. A taxonomy and a comprehensive survey of new drugs discovery is contained in Csermely et al. [8].

An important role in the development of new drugs is given by the methods predicting drug-target interactions (DTI). Traditionally researchers have focused their attention on the development of drugs acting only on a specific protein family. On the other hand the more recent poly-pharmacology approach [9] combines actions of drugs and related multiple targets. The knowledge of these targets is fundamental to find out alternative applications (drug repositioning) as well as to identify side effects $[10,11]$. Despite all such efforts, today many interactions are still unknown and in situ experiments are too costly and time-consuming to be used as the sole strategy.

Various techniques to solve such a problem have been proposed [6,12-16]. In particular, the naive application [17] of the recommendation algorithm developed in Zhou et al. [18] has shown extremely promising results. In Alaimo et al. [19] an extension of the above method with the addition of domain-tuned knowledge led to the definition of the DT-Hybrid algorithm, combining bipartite networks projection and network resources transfer. The process is driven by $2 \mathrm{D}$ drug structural similarity, and target sequential similarity. The basic idea is that structurally similar drugs tend to have analogous behavior in similar proteins.

Another DTI prediction software is STITCH 4.0 [20-23]. It is a database of multi-species protein-chemical interactions, which, not only integrates many experimentally validated and manually curated data sources, but also provides a technique to compute predictions based on text-mining in research articles and analysis of chemical structures. The database combines this information to get an overall score that indicates the degree of confidence of the interaction. However, it can only be applied to the data in their internal database, and users can only search within such data or download the entire list of predictions, rather than providing their own compounds and/or proteins. Moreover, STITCH 4.0 does not compute drugs targeting combinations.

In addition, nowadays, research has been focused on new strategies exploiting metabolic and signaling networks since they show properties for the treatment of infection and cancer diseases. Graph searching algorithms help to exploit such data [24-29]. A metabolic pathway describes a series of chemical reactions occurring within a cell, in which enzymes catalyze the modification of an initial metabolite to form another product. A pathway may include biochemical reactions, complex assembly, transport events, catalysis events and physical interactions involving metabolites, namely proteins, DNA, RNA, small molecules and complexes. The set of metabolic pathways forms the metabolic network of a cell, which is part of its complex system, together with signaling pathways, representing the response to external signals, and other physical processes. Enzymes catalyzing a single reaction are usually essential [30], however related metabolites are frequently involved in several different pathways. Consequently, analyzing metabolic networks helps to identify interesting entities, such as: metabolites used as disease biomarkers [31,32]; or choke points (reactions that consume or produce a certain metabolite) $[33,34]$, whose inhibition may cause lethal deficiencies or toxic accumulation of metabolites. Motivated by the success of manual pathway analysis, a pathway-based approach was proposed in Li et al. [35-37] where a causal drug-to-disease network is built by taking advantage of expert-curated biological knowledge including drug targets, pathways and disease downstream genes. Since, many human diseases cause metabolic deficiencies, the enzymes involved in such essential reactions can be considered promising drug targets [38-41]. Due to the complexity of diseases, the development of multi-target drugs $[42,43]$ or drug combinations may be considered crucial. 
The choke point analysis, the comparison of metabolic networks of pathogenic and non-pathogenic strains and the load point analysis, may improve effective combinations prediction. These commonly consist in the identification of nodes having a high ratio of incident k-shortest paths $[44,45]$. On the other hand, it has been shown that co-targeting of crucial pathway points [46-49] is efficient against drug resistances both in anti-infective [50] and anti-cancer $[51,52]$ strategies. Two relevant examples are RAS and Survivin associated diseases.

The RAS oncogenic mutations occur in human cancer quite frequently [53]. Therapeutic approaches based on the inhibition of RAS-mediated signalling have resulted ineffectively mainly due to the drug's toxicity. On the other hand, mutations of RAS, cause high levels of drug resistance. To overcome such shortcomings, Nussinov et al. [54] suggests an approach, inspired by Holzapfel et al. [55], based on drug combinations on parallel pathways.

The Survivin protein is linked to multiple pathways of cellular homeostasis [56]. These seem to confer to cancer cells a great plasticity, proliferation capability and resistance to death. Moreover, a large number of molecules, regulators, transcriptional networks and modifiers are directly or indirectly involved in Survivin related networks. Therefore, approaches considering Survivin in isolation and based on single-protein inhibitors [57] appear to be unsatisfactory. The development of drugs based on pathways inhibitors [58] exploiting the connectivity maps of Survivin to multiple signaling circuits [4] may result in a more effective approach.

An important application of biological network analysis concerns drug repositioning, a central task in new treatments discovery $[13,14,59,60]$. In Jahchan et al. [61] and Smith et al. [62], a pathway enrichment analysis in combination with gene expression profiles to explore new applications of existing drugs has been exploited. In Pan et al. [63], sixteen FDA-approved drugs were studied in order to understand their clinical functions through pathway analysis. Targets interacting or affected by the investigated drugs were extracted by mining public databases. Pathways having a co-occurrence of such targets were ranked through a p-value. Although directly and indirectly correlated drug targets were identified, important limitations arose due to relevant pathway retrieval and unknown targets finding.

In this paper, we propose DT-Web a software resource accessible via web at [64], using known sources, such as DrugBank [65-67] and PathwayCommons [68], in connection with DT-Hybrid [19] recommendation algorithm. It implements two analysis tools relying on networks: the first one uses a bipartite drug-target interaction network to predict novel high confidence DTIs, while the second one uses a multi-drug, multi-target, multi-pathway approach to guide the early stages of experimental analysis in drug combinations studies. In fact, even if researches can relay on public-domain databases of drug combination references, such as Liu et al. [69], there is still a lack of systematic computational approaches, and often several possible drug combinations are disclaimed by expert knowledge and verified via clinical trials. Motivated by the success of network-based approaches, our multi-purpose pathway analysis aims to provide a limited set of candidate drugs both for drug repositioning and combination, that can be directly evaluated by the experts or combined with other methods.

\section{Construction and content}

The core of DT-Web has been developed in R and Java, while its front-end consists of a web interface developed in PHP with the backing of a MySQL database to store persistent information. We collected only drugs, targets and pathways for the Homo Sapiens species, and designed our methodology accordingly. DT-Web offers two major functionalities: (i) Drug-Target Interaction Prediction: the integration of Drug-Bank with DT-Hybrid to provide a comprehensive database of drugs, and their interactions with proteins (targets, enzymes, transporters, or carriers), either experimentally validated or predicted by DTI network inference; (ii) Multi-Purpose Pathway Analysis: the integration of DrugBank, DT-Hybrid, and PathwayCommons to aid the experimental phase in drug combination studies by searching for drugs simultaneously acting on multiple targets.

\section{Drug-target interaction prediction}

In order to extend our knowledge base, predictions are not limited to small molecules as in Alaimo et al. [19] rather extended to biotech drugs using an appropriate similarity measure based on their synthetic amino-acidic sequence, if available.

In order to search for alternative predictions, DT-Web allows uploading users own data. These may be processed by a $D T$-Hybrid based pipeline. Statistically reliable results will be returned. To guarantee data safety DT-Web assigns to each prediction a random identifier, which is required to retrieve the outcome. More precisely DT-Web actions are summarized below:

\section{Drug-target interactions prediction pipeline}

Step 1. Through an appropriate form, users can upload a DTI network equipped with a similarity matrix for each pair of drugs and targets. DT-Hybrid tuning parameters can be freely modified when this is requested.

Step 2. Users data are then checked to avoid incorrect format, and the DT-Hybrid algorithm is applied to obtain an initial list of predictions for each drug.

Step 3. Each target in the DTI network is mapped to an Entrez identifier in order to annotate each node with 
a set of GO terms. Next, for each pair of terms, we compute a similarity measure based on the node distance in the ontology DAG (Directed Acyclic Graph). Notice that, such a DAG has been previously modified to assign a value to each distance by connecting all the root nodes in the original DAG to a new single dummy root node.

Step 4. Finally, for each drug-target predicted pair, we calculate a correlation measure as the maximum similarity between ontological terms of validated targets and predicted ones. Then, for each drug, we select subsets of predicted targets with increasing minimum correlation, computing, each time, a p-value using a hyper-geometric distribution. Such subsets are filtered to return only those minimizing p-values in each drug. The p-values are computed as follows. Let $M(i, j)$ be the $j$-th subset of predicted targets for drug $i, m$ be the number of targets and $q(i, j)$ be the number of targets having a correlation greater than the minimum computed in $M(i, j)$. The p-value, $\mathrm{p}(M(i, j))$, is the probability of drawing by chance $|M(i, j)|=k(i, j)$ terms whose correlation is greater than the observed minima. An hyper-geometric distribution is used to compute such a value as:

$$
p(M(i, j))=\frac{\left(\begin{array}{l}
q(i, j) \\
k(i, j)
\end{array}\right)\left(\begin{array}{l}
m-q(i, j) \\
k(i, j)-k(i, j)
\end{array}\right)}{\left(\begin{array}{c}
m \\
k(i, j)
\end{array}\right)}=\frac{\left(\begin{array}{l}
q(i, j) \\
k(i, j)
\end{array}\right)}{\left(\begin{array}{c}
m \\
k(i, j)
\end{array}\right)}
$$

The p-value is used to provide a quality score to the association between predicted and validated targets of a single drug. No multiple test correction is applied, as each reported $\mathrm{p}$-value is considered independent from the others.

\section{Multi-purpose pathway analysis}

The aim is to discover the minimal set of drug targets that are able to affect a user-specified set of genes in a multi-pathway environment. The distances among such targets and user genes are limited to a given range in order to minimize drug side effects. The set of validated drug targets is extended with the DT-Hybrid predictions, along with their score to give a measure of confidence on each prediction.

The implemented pipeline has been divided into two main phases. The first phase is performed off-line and kept up-to-date whenever the DT-Web database is synchronized with the latest version of DrugBank. The second one is performed on-line and responses to the pasting of a list of genes through the submission form.

\section{Off-line database building pipeline}

The calculation of a multi-pathway environment requires huge computational resources and it is a time-consuming task. Because of this, the construction of such an environment, consisting of merging the Homo sapiens metabolic and signaling pathways contained in Reactome [70] and PID [71], is done off-line through a proprietary Java module, and stored in our database. The steps are the following.

Step 1. We retrieve all pathways by downloading BioPax [72] level $3 X M L$ files from the Pathway-Commons web service, using PC2 [73] for the remote connection to the public database.

Step 2. For each pathway, we first normalize entity names if these exist (i.e. symbolic names for proteins, such as BRCA1), otherwise we consider the BioPax entity reference IDs.

Step 3. Subsequently, we collapse all nodes representing the same biological entity (protein sub-units or the same protein in different cell locations) in a single node, and we map them to the DrugBank database. Edge directions are kept as they are in the input network, except for those which connect a complex to a constituent protein that are made undirected.

Step 4. The entire set of retrieved pathways is merged into a single global network by mapping nodes and edges using their names and interaction types, respectively.

Step 5. Finally, to control the combinatorial explosion of such data, we store only directed shortest paths between proteins that lie at a distance of, at most, 9 edges. Moreover, since a path could contain edges belonging to different pathways, we decided to store for each edge the list of pathways where it appears. We, also, store the mapping to DrugBank database computed at step 3.

\section{Real-time prediction pipeline}

Step 1. The user provides a list of genes (names in HGNC format, or Uniprot Accession Number, or Entrez Gene Id, or HGNC Id, or Ensembl Gene Id) through a web interface. He can also set the ranges $(\mathrm{min} / \mathrm{max})$ for distances between drug targets and user-provided genes Direct-Indirect Range, or between each pair of drug targets Pair Range.

Step 2. Users' data are thus filtered to remove all proteins that are not present in our database. If the filtered list is not empty, a search is performed in our multipathway environment. The task selects all proteins which are at distance within the Direct-Indirect Range specified by the user.

Step 3. Each protein is then mapped in DrugBank, and those targeted by at least one drug are selected as a preliminary list of targets. Such a list is further filtered by removing all pairs of targets which are outside of the user-specified Pair Range.

Step 4. Next, by applying Chvatal et al. [74], we quickly compute an approximation of the minimum list of targets needed to reach all the user-specified genes. 

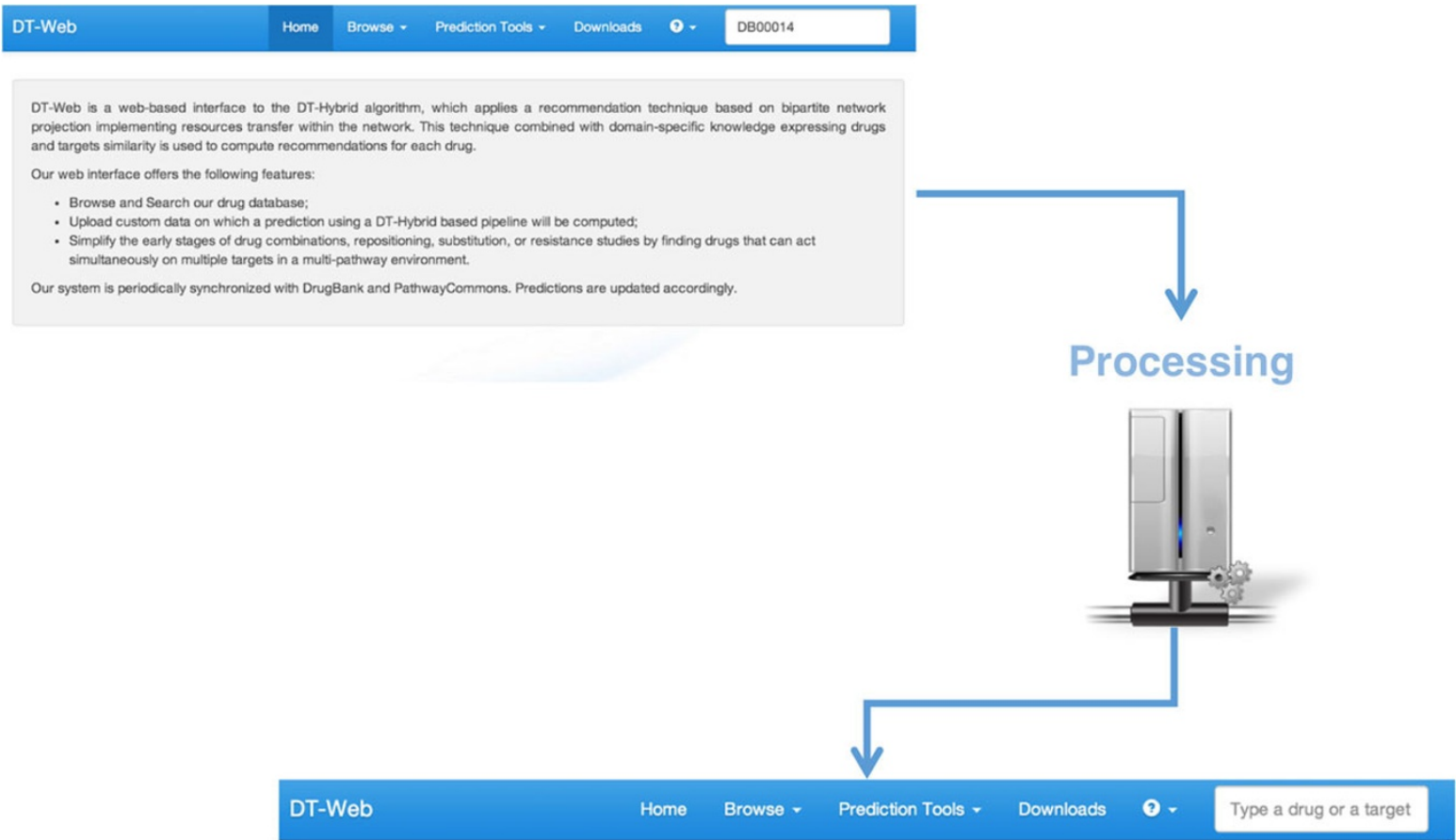

Result for: DB00014

Drug Targets 2 Enzymes 0 Transporters 0

\begin{tabular}{|c|c|}
\hline \multicolumn{2}{|l|}{ Identification } \\
\hline Name & Goserelin \\
\hline $\begin{array}{l}\text { Accession } \\
\text { Number }\end{array}$ & DB00014 (BIOD00113, BTD00113) \\
\hline Type & small molecule \\
\hline Description & $\begin{array}{l}\text { Goserelin is a synthetic hormone. In men, it stops the production of the hormone testosterone, which may stimulate the } \\
\text { growth of cancer cells. In women, goserelin decreases the production of the hormone estradiol (which may stimulate the } \\
\text { growth of cancer cells) to levels similar to a postmenopausal state. When the medication is stopped, hormone levels return } \\
\text { to normal. }\end{array}$ \\
\hline \multicolumn{2}{|l|}{ 2D structure } \\
\hline MOL structure & View structure \\
\hline Molecular Weight & 1269.4105 \\
\hline Groups & approved \\
\hline
\end{tabular}

Figure 1 DT-Web Search Example. Once the user provides a query (either a part of the name or the accession number of a drug/target), DTWeb finds all the matching records in the internal database and returns them to the user in a page containing all the information requested. If all the records are drugs, the user will also see, if available, their two-dimensional structures, and a list of all targets, enzymes, transporters, carriers, and predicted targets, which have been computed through DT-Hybrid. Here user searched for DB00014 


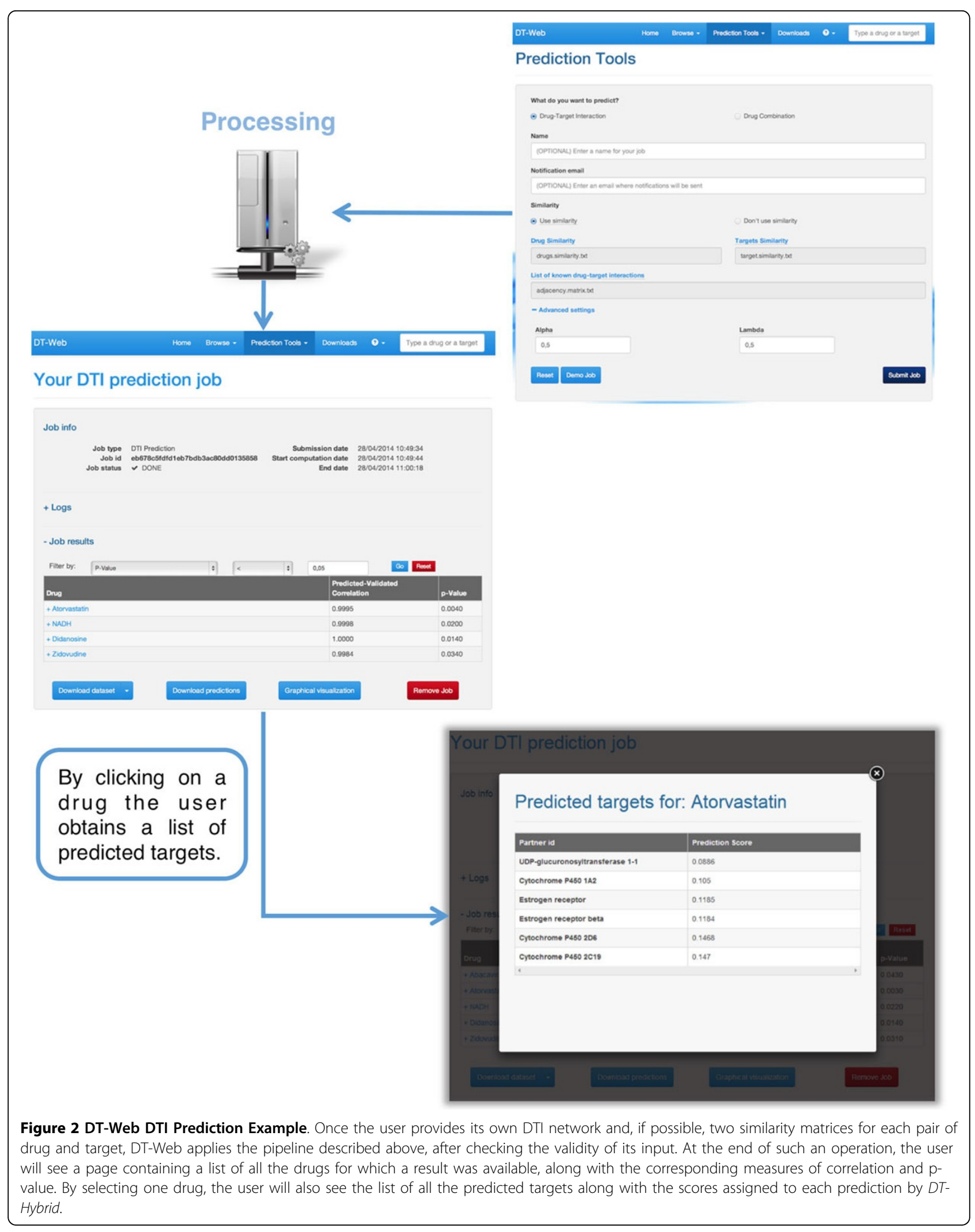


Step 5. Finally, the list of all targets calculated in step 3, and each associated drug (experimentally validated or predicted), is returned to the user, along with the minimum set computed in step 4 .

\section{Utility and discussion}

DT-Web is an effective and user-friendly system that provides a web interface to visualize information on drugs and predicted targets, and to simplify the earlier experimental phases of drug combination studies by applying a multi-drug, multi-target, multi-pathway approach. All this is provided along with a constantly updated database containing the main and most reliable information from DrugBank. In addition, with the ability to upload their own data, our software does not limit the user to use only pre-computed information.

We, also, compared the prediction scores generated by STITCH 4.0 and DT-Web using a set of validated interactions taken from DrugBank. The analysis showed that the knowledge (i.e. experiments, structures, text-mining) given as input to STITCH 4.0 generate a distribution of scores which resulted bimodal and piled either on a low or a up range. On the other hand, DT-Web, which predictions scores are based on structure similarities and network inferences showed a Gaussian behavior. This difference is justifiable by both the kind of input knowledge and inference methods the two systems use.

To use the integrated database, user provides one or more keywords (see our website [64] for a more detailed list of searchable information). The results will be returned on a page, which, as described in Figure 1, contains a list of the main information extracted from DrugBank, experimentally verified targets, enzymes, transporters, and carriers (along with papers providing such experimental evidence), and the predictions calculated by DT-Hybrid.

To take advantage of the DTI prediction pipeline on his own data, the user can choose the "Drug-Target Interaction" job in the "Prediction Tools" menu of our web interface. He will be able to upload his own data (format information and examples are available on our website [64]), which, after checking to ensure that the format is correct, will be analyzed with our pipeline. When data is submitted, DT-Web automatically

\section{DT-Hybrid Web \\ Home Browse - Prediction Tools - Downloads 0 \\ Search}

\section{DTI Graph Visualization}

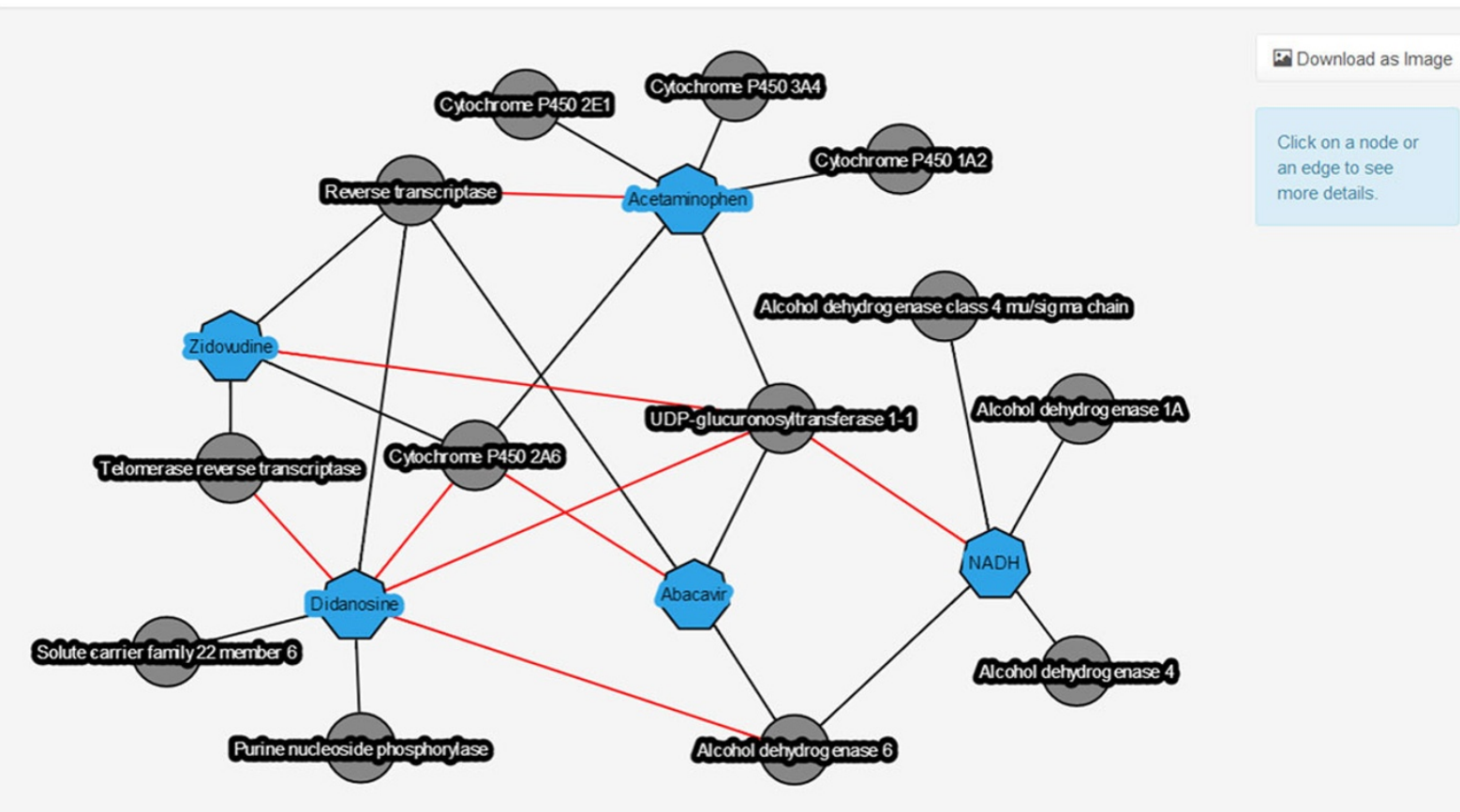

Figure 3 DT-Web DTI network view. A DTI network after applying our prediction pipeline. Each node represents a drug (blue heptagon) or a target (gray circle), while each edge represents a drug-target interaction (user-provided ones in black, predictions in red). 


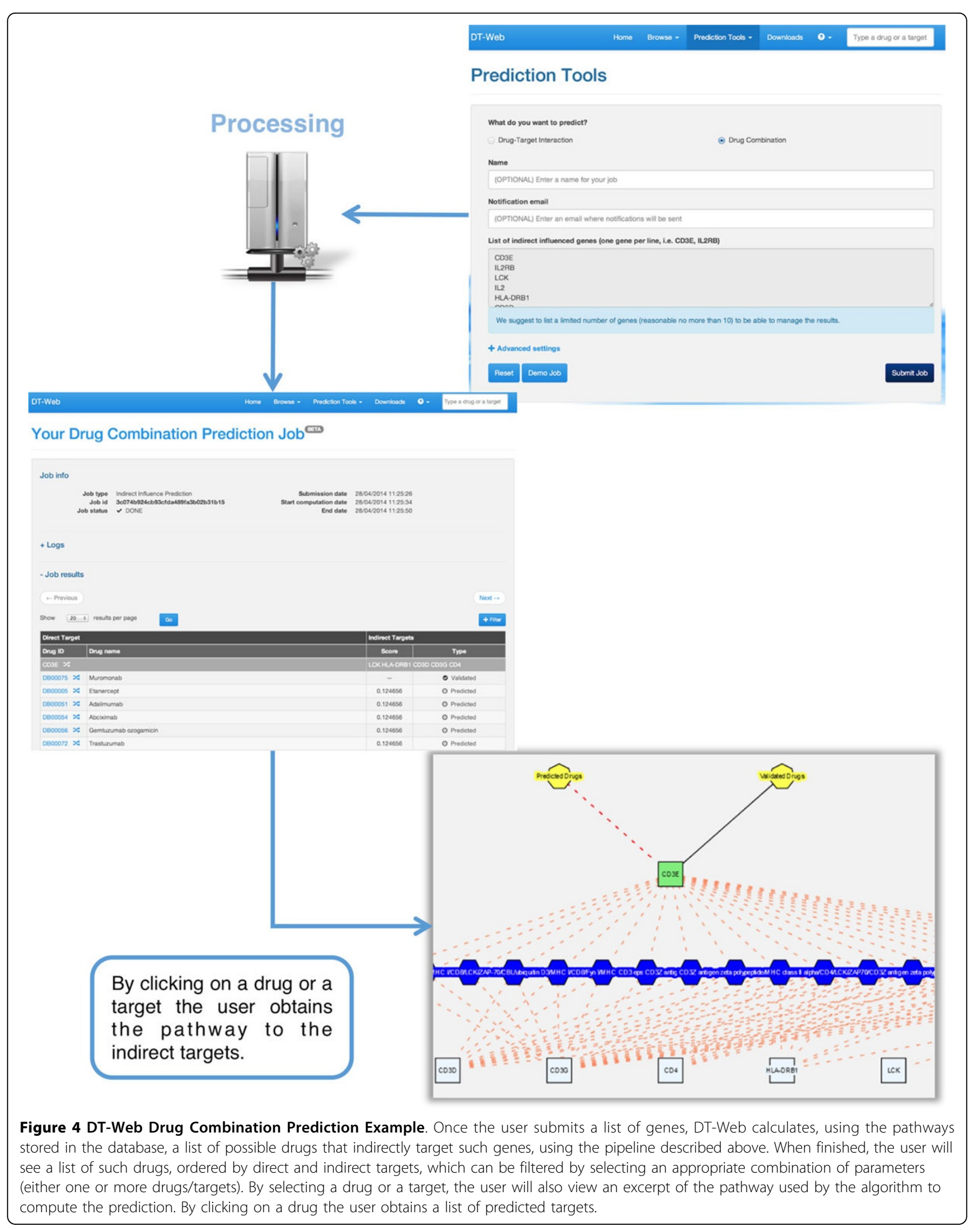


generates a temporary page (see Figure 2) that allows to check the job status and, when ready, view results. To avoid long waits, an e-mail address, where a notification will be sent as soon as the results are available, can be provided. When viewing predictions, the user can apply filters based either on p-value or correlation by choosing an appropriate threshold. He will also be able to download a file containing all the results in text format, or visualize a graphical representation of the resulting DTI network (see Figure 3).

To take advantage of our multi-purpose pipeline, the user can choose the "Drug Combination" job in the "Prediction Tools" menu. There, he can run the enriched analysis by indicating a set of indirectly influenced genes. Once the analysis starts, DT-Web automatically generates a temporary page (see Figure 4) that allows to check the job status and, if ready, view results. Once the analysis is performed, the results page will show the list of reachable targets and their interacting drugs. For each drug-target pair the DT-Hybrid prediction score is shown if the interaction does not come from a validated source. A list of filters can be applied to the results. The list of found drugs can be filtered by selecting only some of them as well as the drug-target interaction type, validated or predicted. Moreover, it is also possible to apply a filter on the set of found targets. For each target and drug, the user can visualize an interactive sub-network centered on the given entity. Additional information is available by clicking on the visualized elements, for example, the list of residing pathways can be visualized by clicking on each edge.

\section{Use cases}

Users access DT-Web via the web interface where drugs and targets can be retrieved using a simple search engine. Furthermore, users can provide their own data to compute DTI predictions or simplify the early development stages in drug combination or repositioning studies. The algorithm is also available for download under Creative Commons license. This allows users to use DT-Web for large-scale studies, not suitable for our web environment. Examples illustrating our DTI prediction and multi-purpose pipelines follow.

DT-Hybrid has been used, in connection with other algorithms, to associate Simvastatin and Ketoconazole drugs to breast cancer treatment [75]. Our multipurpose pipeline, being a new software, has not yet been used for the intended studies. That is why we used our methodology to predict the combination of Propofol and Sevoflurane whose additive action produces consciousness and movement to skin incision during general anesthesia [76]. Both drugs interact with the $\mathrm{GABA}_{\mathrm{A}}$ receptor. Propofol is a potentiator of the $\beta 2$ subunit (GABRB2) of $G A B A_{A}$, while Sevoflurane is an agonist of the $\alpha 1$ subunit (GABRA1) of $\mathrm{GABA}_{\mathrm{A}}$ with its binding site between both subunits [77]. Probably is such a location which hinders agonist activity, thereby producing mutually substitutable actions [78].

\section{Conclusions}

The prediction of novel drug-target interactions is a fundamental process in order to reduce the costly and timeconsuming phases of drug discovery and design. As a matter of fact, knowing the possible unknown effects on the proteome of a drug can be extremely useful in understanding its true potential or predicting side effects.

Other studies, such as drug repositioning, drug combinations or substitutions, help to eliminate the need to develop new drugs. Drug repositioning studies exploit existing drug for new purposes that go beyond the original ones, while drug combination studies try to modify or intensify the overall effect of two or more drugs by administering them together. Similarly, drug substitutions studies try to replace drugs having significant side effects with others aiming to reduce them. For this purpose, a tool that guides the early stages of the experimental process can significantly reduce the time and costs associated therewith.

This is the context of DT-Web. Its main goal is to provide a simple system that allows a user to quickly browse predictions of probable novel DTI, to produce new ones from their own data, or to simplify the experimental studies described above. All this by using a database obtained combining a new valuable resource $D T$ Hybrid with data extracted from Drug-Bank and PathwayCommons. Finally, all the results can be downloaded in text format or viewed on-line through the help of interactive graphical interfaces that simplify their understanding.

\section{List of abbreviations used \\ DTI: Drug-Target Interactions \\ PID: The pathway interaction database \\ DAG: Directed acyclic graph}

\section{Competing interests}

The authors declare that they have no competing interests.

\section{Authors' contributions}

RG and AP conceived, developed and coordinated the research. SA and VB designed and developed the system. DC contributed to implementation aspects. SA, VB, AF, RG and AP analyzed results and wrote the paper.

\section{Acknowledgements}

RG, AP and AF were partially supported by "Progetto di Ricerca GNCS INDAM 2014".

\section{Declarations}

Publication of this article has been funded by PON 2007-2013 grant, SIGMA PON01 00683 - CUP B61H11000380005.

This article has been published as part of BMC Systems Biology Volume 9 Supplement 3, 2015: Proceedings of the Italian Society of Bioinformatics (BITS): Annual Meeting 2014: Systems Biology. The full contents of the 
supplement are available online at http://www.biomedcentral.com/ bmcsystbiol/supplements/9/S3.

\section{Authors' details}

'Department of Mathematics and Computer Science, University of Catania, Viale A. Doria 6, Catania, Italy. ${ }^{2}$ Department of Clinical and Experimenta Medicine, University of Catania, Viale A. Doria 6, Catania, Italy. ${ }^{3}$ Department of Computer Science, University of Verona, Strada le Grazie 15, Verona, Italy. ${ }^{4}$ Correspondence can be also addressed to giugno@dmi.unict.it.

Published: 1 June 2015

\section{References}

1. Chong CR, Sullivan DJ Jr: New uses for old drugs. Nature 2007 448(7154):645-646.

2. Phatak SS, Zhang S: A novel multi-modal drug repurposing approach for identification of potent ack1 inhibitors. Pacific Symposium on Biocomputing 2013, 29-40.

3. Iorio F, Bosotti R, Scacheri E, Belcastro V, Mithbaokar P, Ferriero R, et al: Discovery of drug mode of action and drug repositioning from transcriptional responses. Proceedings of the National Academy of Sciences 2010, 107(33):14621-14626.

4. Lamb J: The connectivity map: a new tool for biomedical research. Nature Reviews Cancer 2007, 7(1):54-60.

5. Cokol M, lossifov I, Weinreb C, Rzhetsky A: Emergent behavior of growing knowledge about molecular interactions. Nature biotechnology 2005 , 23(10):1243-1248.

6. Yildirim MA, Goh Kl, Cusick ME, Barabási AL, Vidal M: Drug-target network Nature Biotechnology 2007, 25(10):1119-1126

7. Iyer $P, H u Y$, Bajorath J: SAR monitoring of evolving compound data sets using activity landscapes. J Chem Inf Model 2011, 51(3):532-540.

8. Csermely P, Korcsmáros T, Kiss HJ, London G, Nussinov R: Structure and dynamics of molecular networks: A novel paradigm of drug discovery: A comprehensive review. Pharmacol Ther 2013, 138(3):333-408.

9. Hopkins AL: Network pharmacology: the next paradigm in drug discovery. Nature Chem Biol 2008, 4(11):682-690.

10. Ashburn $\Pi$, Thor $K B$ : Drug repositioning: identifying and developing new uses for existing drugs. Nat Rev Drug Discov 2004, 3(8):673-683.

11. Boguski MS, Mandl KD, Sukhatme VP: Drug discovery. Repurposing with a difference. Science 2009, 324(5933):1394-1395.

12. Yamanishi Y, Araki M, Gutteridge A, Honda W, Kanehisa M: Prediction of drug-target interaction networks from the integration of chemical and genomic spaces. Bioinformatics 2008, 24(13):i232-i240.

13. Dudley JT, Sirota M, Shenoy M, Pai RK, Roedder S, Chiang AP, et al: Computational repositioning of the anticonvulsant topiramate for inflammatory bowel disease. Sci Transl Med 2011, 3(96):96ra76.

14. Sirota M, Dudley JT, Kim J, Chiang AP, Morgan AA, Sweet-Cordero A, et al: Discovery and preclinical validation of drug indications using compendia of public gene expression data. Sci Transl Med 2011 3(96):96ra77.

15. van Laarhoven T, Nabuurs SB, Marchiori E: Gaussian interaction profile kernels for predicting drug-target interaction. Bioinformatics 2011, 27(21):3036-3043.

16. Mei JP, Kwoh CK, Yang $P$, Li XL, Zheng J: Drug-target interaction prediction by learning from local information and neighbors. Bioinformatics 2013, 29(2):238-245.

17. Cheng F, Liu C, Jiang J, Lu W, Li W, Liu G, et al: Prediction of drug-target interactions and drug repositioning via network-based inference. PLOS Comput Biol 2012, 8(5):e1002503.

18. Zhou T, Ren J, Medo M, Zhang YC: Bipartite network projection and personal recommendation. Physical Review E 2007, 76(4):046115.

19. Alaimo S, Pulvirenti A, Giugno R, Ferro A: Drug-target interaction prediction through domain-tuned network-based inference. Bioinformatics 2013, 29(16):2004-2008.

20. Kuhn M, von Mering C, Campillos M, Jensen L, Bork P: STITCH: interaction networks of chemicals and proteins. Nucleic Acids Research 2008 36(Database issue):D684-D688.

21. Kuhn M, Szklarczyk D, Franceschini A, Campillos M, von Mering C, Jensen $L$, et al: STITCH 2: an interaction network database for small molecules and proteins. Nucleic Acids Research 2010, 38(Database issue): D552-D556.
22. Kuhn M, Szklarczyk D, Franceschini A, von Mering $C$, Jensen $L$, Bork $P$ : STITCH 3: zooming in on protein-chemical interactions. Nucleic Acids Research 2012, 40(Datbase issue):D876-D880.

23. Kuhn M, Szklarczyk D, Pletscher-Frankild S, Blicher TH, von Mering C, Jensen LJ, Bork P: STITCH 4: integration of protein-chemical interactions with user data. Nucleic Acids Research 2014, 42(Database issue):D401-D407.

24. Micale G, Pulvirenti A, Giugno R, Ferro A: GASOLINE: a greedy and stochastic algorithm for optimal local multiple alignment of interaction networks. PLOS ONE 9(6):e98750.

25. Giugno R, Bonnici $V$, Bombieri N, Pulvirenti A, Ferro A, Shasha D: GRAPES: a software for parallel searching on biological graphs targeting multi-core architectures. PLOS ONE 2013, 8(10):e76911.

26. Bonnici V, Giugno R, Pulvirenti A, Shasha D, Ferro A: A subgraph isomorphism algorithm and its application to biochemical data. BMC Bioinformatics 2013, 14(Suppl 7):S13

27. Ferro A, Giugno R, Mongiovì M, Pulvirenti A, Skripin D, Shasha D: GraphFind: enhancing graph searching by low support data mining techniques. BMC Bioinformatics 2008, 9(Suppl 4):S10.

28. Mongiovi M, Di Natale R, Giugno R, Pulvirenti A, Ferro A, Sharan R: A setcover-based approach for inexact graph matching. Journal of Bioinformatics and Computational Biology 2010, 8:199-218.

29. Di Natale R, Ferro A, Giugno R, Mongiovi M, Pulvirenti A, Shasha D: SING: Subgraph search in non-homogeneous graphs. BMC Bioinformatics 2010, 11:96.

30. Nam H, Lewis NE, Lerman JA, Lee DH, Chang RL, Kim D, Palsson BO: Network context and selection in the evolution to enzyme specificity. Science 2012, 337(6098):1101-1104.

31. Fan TW, Lorkiewicz PK, Sellers K, Moseley HN, Higashi RM, Lane AN: Stable isotope-resolved metabolomics and applications for drug development. Pharmacol Ther 2012, 133(3):366-391.

32. Shlomi T, Cabili MN, Ruppin E: Predicting metabolic biomarkers of human inborn errors of metabolism. Mol Syst Biol 2009, 5:263.

33. Yeh I, Hanekamp T, Tsoka S, Karp PD, Altman RB: Computational analysis of plasmodium falciparum metabolism: organizing genomic information to facilitate drug discovery. Genome Res 2004, 14(5):917-924.

34. Singh S, Malik BK, Sharma DK: Choke point analysis of metabolic pathways in e. histolytica: a computational approach for drug target identification. Bioinformation 2007, 2(2):68-72.

35. Li J, Lu Z: Pathway-based drug repositioning using causal inference. $B M C$ Bioinformatics 2013, 14(Suppl 16):S3.

36. Li J, Lu Z: A new method for computational drug repositioning using drug pairwise similarity. Bioinformatics and Biomedicine (BIBM), 2012 IEEE International Conference On IEEE; 2012, 1-4.

37. Li Y, Agarwal P: A pathway-based view of human diseases and disease relationships. PloS One 2009, 4(2):e4346.

38. Kim HU, Kim TY, Lee SY: Genome-scale metabolic network analysis and drug targeting of multi-drug resistant pathogen acinetobacter baumannii aye. Molecular BioSystems 2010, 6(2):339-348.

39. Kim HU, Kim SY, Jeong H, Kim TY, Kim JJ, Choy HE, et al: Integrative genome-scale metabolic analysis of vibrio vulnificus for drug targeting and discovery. Molecular Syst Biol 2011, 7:460

40. Kim Y, Kim TK, Kim Y, Yoo J, You S, Lee l, et al: Principal network analysis: identification of subnetworks representing major dynamics using gene expression data. Bioinformatics 2011, 27(3):391-398.

41. Ma H, Goryanin I: Human metabolic network reconstruction and its impact on drug discovery and development. Drug Discovery Today 2008, 13(9-10):402-408.

42. Ma J, Zhang $X$, Ung CY, Chen YZ, Li B: Metabolic network analysis revealed distinct routes of deletion effects between essential and nonessential genes. Molecular BioSystems 2012, 8(4):1179-1186.

43. Barve A, Rodrigues JFM, Wagner A: Superessential reactions in metabolic networks. Proceedings of the National Academy of Sciences 2012, 109(18): E1121-E1130.

44. Fatumo S, Plaimas K, Mallm JP, Schramm G, Adebiyi E, Oswald M, Eils R, König R: Estimating novel potential drug targets of Plasmodium falciparum by analysing the metabolic network of knock-out strains in silico. Infection, Genetics and Evolution 2009, 9(3):351-358.

45. Perumal D, Lim CS, Sakharkar MK: A comparative study of metabolic network topology between a pathogenic and a non-pathogenic bacterium for potential drug target identification. Summit on Translat Bioinforma 2009, 2009:100-104. 
46. Zimmermann GR, Lehar J, Keith CT: Multi-target therapeutics: when the whole is greater than the sum of the parts. Drug Discov Today 2007, 12(1):34-42.

47. Pujol A, Mosca R, Farrés J, Aloy P: Unveiling the role of network and systems biology in drug discovery. Trends Pharmacol Sci 2010, 31(3):115-123.

48. Rosado JO, Henriques JP, Bonatto D: A systems pharmacology analysis of major chemotherapy combination regimens used in gastric cancer treatment: predicting potential new protein targets and drugs. Cur Cancer Drug Targets 2011, 11(7):849-869.

49. Savino R, Paduano S, Preianò M, Terracciano R: The proteomics big challenge for biomarkers and new drug-targets discovery. Int J Mol Sci 2012, 13(11):13926-13948.

50. Bush K, Courvalin P, Dantas G, Davies J, Eisenstein B, Huovinen P, et al: Tackling antibiotic resistance. Nature Reviews Microbiology 2011, 9(12):894-896

51. Kitano H: Biological robustness. Nature Reviews Genetics 2004, 5(11):826-837

52. Logue JS, Morrison DK: Complexity in the signaling network: insights from the use of targeted inhibitors in cancer therapy. Genes Dev 2012, 26(7):641-650.

53. Adjei AA: Blocking oncogenic Ras signaling for cancer therapy. Journal of the National Cancer Institute 2001, 93(14):1062-1074.

54. Nussinov R, Tsai CJ, Mattos C: 'Pathway drug cocktail': targeting Ras signaling based on structural pathways. Trends Mol Med 2013, 19(11):695-704.

55. Holzapfel G, Buhrman G, Mattos C: Shift in the equilibrium between on and off states of the allosteric switch in Ras-GppNHp affected by small molecules and bulk solvent composition. Biochemistry 2012, 51(31):6114-6126.

56. Altieri DC: Survivin, cancer networks and pathway-directed drug discovery. Nature Reviews Cancer 2008, 8(1):61-70.

57. Sawyers C: Targeted cancer therapy. Nature 2004, 432(7015):294-297.

58. van der Greef J, McBurney RN: Innnovation: Rescuing drug discovery: in vivo systems pathology and systems pharmacology. Nature Reviews Drug Discovery 2005, 4(12):961-967.

59. Dudley JT, Deshpande T, Butte AJ: Exploiting drug-disease relationships for computational drug repositioning. Brief Bioinform 2011, 12(4):303-311.

60. Lamb J, Crawford ED, Peck D, Modell JW, Blat IC, Wrobel MJ, et al, et al: The connectivity map: using gene-expression signatures to connect small molecules, genes, and disease. Science 2006, 313(5795):1929-1935.

61. Jahchan NS, Dudley JT, Mazur PK, Flores N, Yang D, Palmerton A, et al: A drug repositioning approach identifies tricyclic antidepressants as inhibitors of small cell lung cancer and other neuroendocrine tumors. Cancer Discovery 2013, 3(12):1364-1377.

62. Smith SB, Dampier W, Tozeren A, Brown JR, Magid-Slav M: Identification of common biological pathways and drug targets across multiple respiratory viruses based on human host gene expression analysis. PLOS One 2012, 7(3):e33174

63. Pan $Y$, Cheng T, Wang Y, Bryant SH: Pathway analysis for drug repositioning based on public database mining. Journal of chemical information and modeling 2014, 54(2):407-418.

64. DT-Hybrid., Web. http://alpha.dmi.unict.it/dtweb/.

65. Knox C, Law V, Jewison T, Liu P, Ly S, Frolkis A, et al: Drugbank 3.0: a comprehensive resource for 'omics' research on drugs. Nucleic Acids Res 2011, 39(Database issue):D1035-D1041.

66. Wishart DS, Knox C, Guo AC, Cheng D, Shrivastava S, Tzur D, et al: DrugBank: a knowledgebase for drugs, drug actions and drug targets. Nucleic Acids Res 2008, 36(Database issue):D901-D906.

67. Wishart DS, Knox C, Guo AC, Shrivastava S, Hassanali M, Stothard P, et al: DrugBank: a comprehensive resource for in silico drug discovery and exploration. Nucleic Acids Res 2006, 34(Database issue):D668-D672.

68. Cerami EG, Gross BE, Demir E, Rodchenkov I, Babur Ö, Anwar N, et al: Pathway Commons, a web resource for biological pathway data. Nucleic Acids Res 2011, 39(Database issue):D685-D690.

69. Liu Y, Hu B, Fu C, Chen X: DCDB: drug combination database. Bioinformatics 2010, 26(4):587-588.

70. Croft D, Mundo AF, Haw R, Milacic M, Weiser J, Wu G, et al: The reactome pathway knowledgebase. Nucleic Acids Res 2014, 42(Database issue): D472-D477.
71. Schaefer CF, Anthony K, Krupa S, Buchoff J, Day M, Hannay T, Buetow KH: PID: the pathway interaction database. Nucleic Acids Res 2009, 37(Database issue):D674-D679.

72. Demir E, Cary MP, Paley S, Fukuda K, Lemer C, Vastrik I, et al: The BioPAX community standard for pathway data sharing. Nature Biotechnology 2010, 28(9):935-942.

73. cPath2., http://www.pathwaycommons.org/pc2/.

74. Chvatal V: A greedy heuristic for the set-covering problem. Mathematics of Operations Research 1979, 4(3):233-235.

75. Jin G, Wong STC: Toward better drug repositioning: prioritizing and integrating existing methods into efficient pipelines. Drug Discovery Today 2014, 19(5):637-644.

76. Harris RS, Lazar O, Johansen JW, Sebel PS: Interaction of propofol and sevoflurane on loss of consciousness and movement to skin incision during general anesthesia. Anesthesiology 2006, 104(6):1170-1175.

77. Sigel E: Mapping of the benzodiazepine recognition site on GABA(A) receptors. Current topics in medicinal chemistry 2002, 2(8):833-839.

78. Jia J, Zhu F, Ma X, Cao ZW, Li YX, Chen YZ: Mechanisms of drug combinations: interaction and network perspectives. Nature Reviews Drug Discovery 2009, 8(2):111-128.

doi:10.1186/1752-0509-9-S3-S4

Cite this article as: Alaimo et al.: DT-Web: a web-based application for drug-target interaction and drug combination prediction through domain-tuned network-based inference. BMC Systems Biology 2015 9(Suppl 3):S4.

\section{Submit your next manuscript to BioMed Central and take full advantage of:}

- Convenient online submission

- Thorough peer review

- No space constraints or color figure charges

- Immediate publication on acceptance

- Inclusion in PubMed, CAS, Scopus and Google Scholar

- Research which is freely available for redistribution

Submit your manuscript at www.biomedcentral.com/submit
C) Biomed Central 